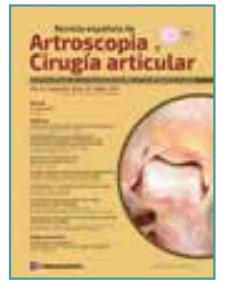

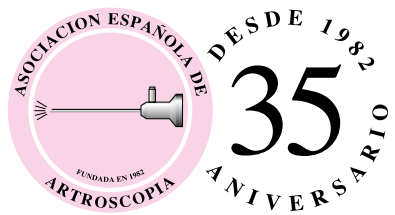

Vol. 24. Especial. Núm. 57. Mayo 2017

ISSN: 2386-3129 (impreso)

2443-9754 (online)

\title{
Original
}

\section{Elección del injerto en la reconstrucción de ligamento cruzado anterior. ¿Existe un injerto ideal?}

\author{
R. Calvo, Z. Anastasiadis, R. Calvo Mena, D. Figueroa \\ Clínica Alemana. Universidad del Desarrollo. Santiago de Chile, Chile \\ Correspondencia: \\ Dr. Rafael Calvo Rodríguez \\ Correo electrónico: rcalvo@alemana.cl
}

Recibido el 12 de abril de 2017

Aceptado el 12 de abril de 2017

Disponible en Internet: mayo de 2017

\section{RESUMEN}

Existen numerosos trabajos sobre diferentes injertos para la reconstrucción del ligamento cruzado anterior. Sin embargo, no hay un acuerdo sobre cuál es el injerto ideal. En el presente artículo revisaremos lo publicado sobre las ventajas y desventajas de cada tipo de injerto, así como sus resultados comparativos. Después de nuestra revisión, no hemos encontrado un consenso sobre qué injerto elegir. Se debe decidir en función de las características del paciente, sus expectativas de recuperación y la vuelta a la actividad deportiva. La mayor controversia existe en pacientes jóvenes y con alto nivel de actividad deportiva.

Palabras clave: Ligamento cruzado anterior. Reconstrucción. Injerto. Deporte. Rodilla.

\section{Introducción}

El mejor entendimiento de la anatomía y de la función del ligamento cruzado anterior (LCA) ha permitido perfeccionar las diferentes técnicas quirúrgicas para su reconstrucción e integración.

\begin{abstract}
Choosing a graft in anterior cruciate ligament reconstruction. Is there an ideal graft?

There are numerous studies in the literature concerning different grafts for the reconstruction of the anterior cruciate ligament, however there is no agreement on the ideal graft. In this article, we will review the reported advantages and disadvantages of each type of graft, as well as their comparative results. According to the evidence, there is no consensus on what graft should be chosen. It must be decided based on the characteristics of the patient, their expectations of recovery and sports return. Young patients, increased level of activity and allograft have been consistently identified as points of greatest controversy.
\end{abstract}

Key words: Anterior cruciate ligament. Repair. Graft. Sport. Knee.

Sin embargo, a pesar del gran número de publicaciones, la elección del injerto "ideal" sigue siendo motivo de debate, así como su implicación en el eventual fracaso del neoligamento. El injerto ideal debe reproducir las propiedades estructurales del LCA normal, debe tener una baja tasa

http://doi.org/10.24129/j.reaca.24e57.fs1704017

FS $\odot 2017$ Fundación Española de Artroscopia. Publicado por Imaidea Interactiva en FONDOSCIENCE ${ }^{\circledR}$ (www.fondoscience.com) Este es un artículo Open Access bajo la licencia CC BY-NC-ND (www.creativecommons.org/licenses/by-nc-nd/4.0/). 
de morbilidad, fácil disponibilidad, baja reacción inmunogénica y proveer además una fijación fuerte para permitir una rehabilitación precoz y exigente ${ }^{(1)}$. Por otro lado, el uso de determinado tipo de injerto debe ser seleccionado según cada paciente en relación con su actividad deportiva y expectativas.

Son pocos los estudios estadísticos que permitan definir una conducta absoluta en relación con la selección del injerto para cada paciente(2). Este artículo tiene por objetivo dar directrices en la selección del injerto para cada tipo de paciente, en función de la evidencia reportada y la experiencia.

\section{Tipos de injerto y sus características}

Existen diversos tipos de injertos (Tabla 1) y su uso tiende a seleccionar determinados injertos para cierto grupo de pacientes, por lo que es importante revisar los resultados de la bibliografía. Por ejemplo, es frecuente asociar el uso de tendón patelar con los deportes de impacto por considerarlo más fuerte o utilizar los tendones flexores en pacientes con menor demanda, por ofrecer una menor morbilidad asociada. También es frecuente considerar el aloinjerto para las revisiones o lesiones multiligamentosas. Pero, ¿tienen una base sólida, con un nivel de evidencia adecuado, estas afirmaciones?

\section{Tendón rotuliano}

El injerto se obtiene del tercio central del tendón patelar con la pastilla ósea de patela y tuberosidad tibial adyacente en los extremos respectivos. Ha sido considerado históricamente cómo el gold standard(3,4) para la reconstrucción del LCA, basado en su extenso uso; al ser uno de los primeros injertos utilizados, es también el que tiene un mayor seguimiento. Sus ventajas radican en la incorporación más temprana de la pastilla ósea (6 semanas) $)^{(5)}$, debido a que la interfase hueso-hueso logra una consolidación mejor que la interfaz tejido blando-hueso de los otros injertos. Además, su fijación es segura, siendo superior a los injertos de partes blandas, alcanzando tanto con tornillos metálicos como bioabsorbibles una resistencia de 552 a $558 \mathrm{~N}^{(6)}$. Ambas caracteristi-

\begin{tabular}{l|l|l}
\hline \multicolumn{2}{c}{$\begin{array}{l}\text { Tabla 1. Clasificación de los diferentes injertos } \\
\text { utilizados* }\end{array}$} \\
\hline \multirow{2}{*}{ Autoinjertos } & Aloinjertos & $\begin{array}{l}\text { Injertos } \\
\text { sintéticos }\end{array}$ \\
\hline \multirow{2}{*}{ Tendón patelar } & Aquiles & Matrices \\
\cline { 2 - 2 } & Tendón patelar & \\
\hline \multirow{2}{*}{$\begin{array}{l}\text { Tendones } \\
\text { isquiotibiales }\end{array}$} & Isquiotibiliales & \multirow{2}{*}{ Stents } \\
\cline { 2 - 2 } & Tendón cuadricipital & \\
\hline \multirow{2}{*}{$\begin{array}{l}\text { Tendón } \\
\text { cuadricipital }\end{array}$} & $\begin{array}{l}\text { Tibial anterior o } \\
\text { posterior }\end{array}$ & \multirow{2}{*}{ Prótesis } \\
\cline { 2 - 2 } & Fascia lata & \\
\hline
\end{tabular}

* En el grupo de autoinjertos consideramos los más ampliamente utilizados

cas permiten una rehabilitación más agresiva y un retorno deportivo más precoz. Otras ventajas comparativas son que no altera la fuerza de los isquiotibiales sanos y que se asocia a un menor costo relacionado con los medios de fijación.

Los principales riesgos descritos ${ }^{(7)}$ para este injerto son la fractura patelar tanto intraoperatoria como después de la cirugía (2,3\%), la fractura de fatiga de la tibia, el daño articular del cartílago patelar y la rotura tendinosa.

Este injerto se ha asociado a un mayor riesgo de dolor en la cara anterior de la rodilla, con una incidencia del 17,4 al 47\% ${ }^{\left({ }^{8}\right)}$ Por otra parte, el autoinjerto de los tendones isquiotibiales presenta dolor en la rodilla en el $11,8 \%^{(9)}$ de los casos y no existe diferencia en el dolor anterior al comparar injerto de tendón patelar con el aloinjerto. Por eso se ha pensado que la incidencia de dolor anterior obedece en cierta medida a un programa de rehabilitación deficiente y no al tipo de injerto. Otras complicaciones descritas ${ }^{(8)}$ incluyen el desarrollo de tendinosis (6\%) y daño de la rama infrapatelar del nervio safeno. Si bien se ha descrito atrofia de cuádriceps tras la cirugía en el $10 \%$ de los $\operatorname{casos}^{(8)}$, otros estudios muestran que la toma del injerto patelar no disminuye la fuerza del músculo cuádriceps en pacientes que siguen un programa de rehabilitación intensiva ${ }^{(10)}$.

Técnicamente, también ofrece algunas dificultades en reconstrucciones anatómicas, donde los túneles son más cortos y este injerto puede ser de mayor longitud, comprometiendo la fijación ósea en el túnel tibial. 


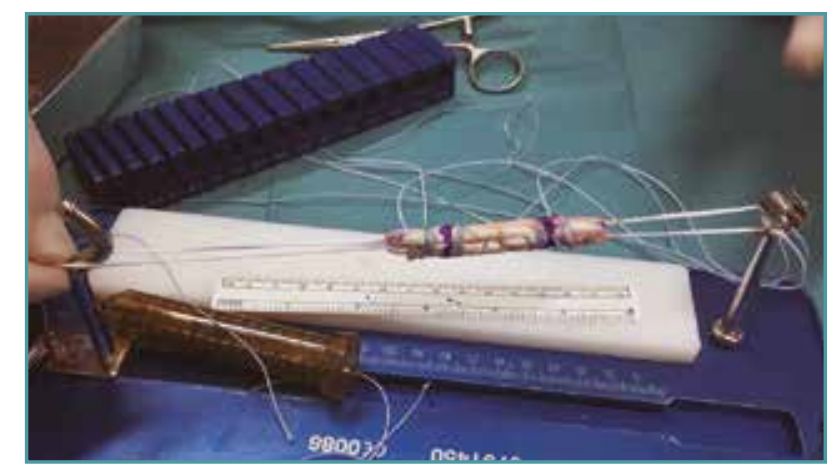

Figura 1. Autoinjerto del tendón de los músculos semitendinoso y gracilis quíntuple. Se obtiene un injerto de mayor diámetro, utilizando un botón cortical para la fijación femoral.

\section{Tendones flexores (isquiotibiales)}

Este injerto se obtiene de los tendones de inserción de los músculos semitendinoso y gracilis. Existe una diversidad de técnicas descritas en cuanto a utilizarlo cuádruple, triple o quíntuple, así como en los sistemas de fijación utilizados para ellos (Figura 1). Sus ventajas están dadas por ser un procedimiento más estético, con mayor resistencia biomecánica del injerto cuádruple ${ }^{(11)}$ (Tabla 2) y menor dolor en la zona dadora. En las reconstrucciones anatómicas ofrece ventajas técnicas a considerar. En relación con sus desventajas, se debe considerar que su diámetro no es predecible, lo que es fundamental dado un mayor riesgo de rerrotura en diámetros inferiores a $8 \mathrm{~mm}$. También la integración del tendón al hueso es mas lenta (12 semanas) y presenta menor resistencia en sus fijaciones, especialmente en el túnel tibial. Se ha demostrado una mayor elongación del injerto, principalmente en mujeres, niños y pacientes hiperlaxos $^{(12)}$. Las complicaciones asociadas a su uso consisten en el daño del nervio safeno y las eventuales complicaciones neurovasculares.

La debilidad de la musculatura isquiotibial residual demuestra que se corrige a partir del tercer mes, sin observarse implicancia en los resultados clínicos ${ }^{(1)}$.

\section{El tendón cuadricipital}

Quizá menos popular que el tendón patelar y los isquiotibiales en nuestro medio, es una alternativa viable para la reconstrucción del LCA. Staubli et al. ${ }^{(13)}$ demostraron las propiedades biomecánicas del tendón y Fulkerson y Langeland ${ }^{(14)}$ vieron buenos resultados iniciales.

El injerto se toma con una pastilla ósea, de $10 \times 20 \mathrm{~mm}$, de la patela proximal y resecando aproximadamente $7 \mathrm{~cm}$ de tendón hacia proximal.

Puede presentar una mayor dificultad técnica, ya que se debe evitar dañar el receso suprapatelar.

El tendón cuadricipital tiene la ventaja de ofrecer una mayor área de sección transversal para la porción intraarticular del injerto, otorgando buenas propiedades biomecánicas. Tiene menor tasa de dolor anterior postoperatorio comparado con el tendón patelar, pero puede generar una cicatriz antiestética ${ }^{(15)}$. Otra desventaja es debilidad muscular postoperatoria, al comprometer el aparato extensor ${ }^{(16)}$. 


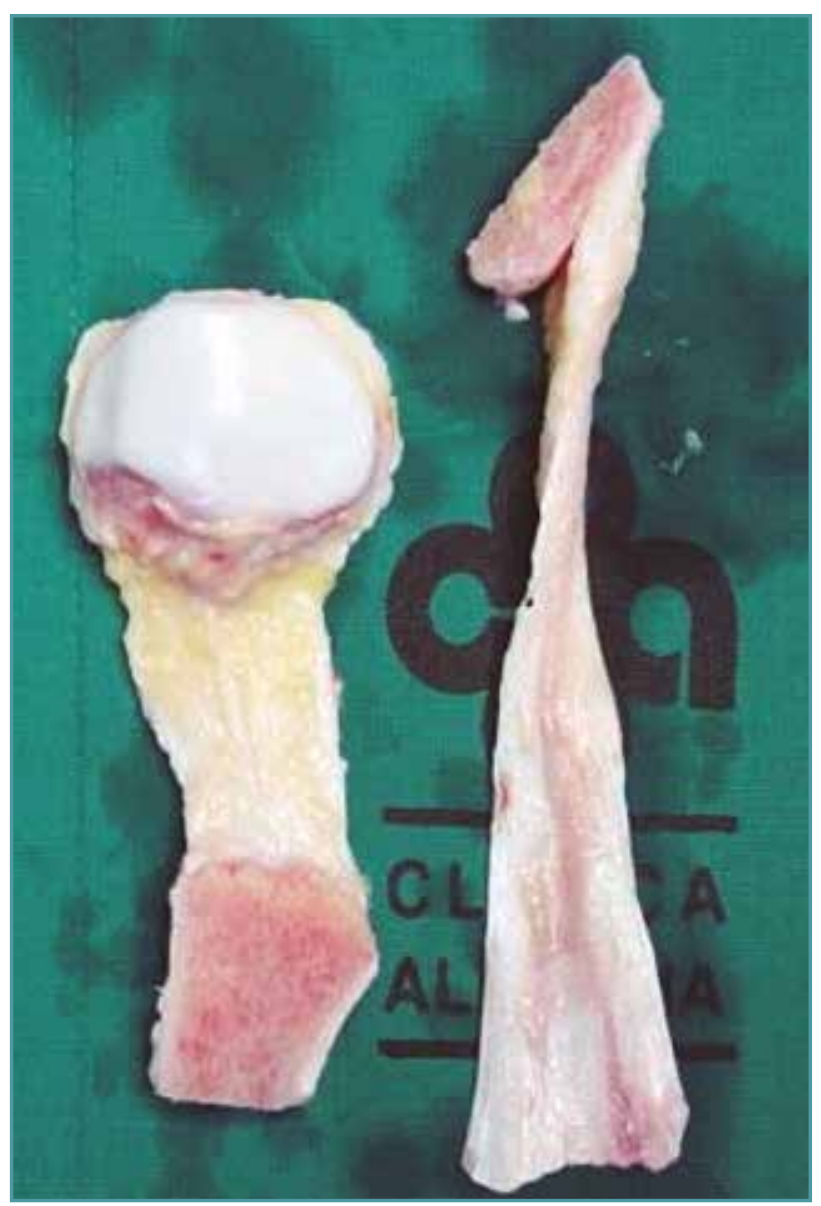

Figura 2. Aloinjertos de tendón patelar y tendón de Aquiles.

\section{Aloinjerto}

La gran ventaja de los aloinjertos es evitar la morbilidad de la zona dadora. Sin embargo, presenta el riesgo potencial de transmisión de enfermedades y, por esta razón, existe un estricto control del tejido cosechado y del historial médico del donante. En la bibliografía solo existe un caso descrito de transmisión del virus de la inmunodeficiencia humana (VIH) y dos casos de transmisión de hepatitis $\mathrm{C}^{(17)}$. El riesgo estimado para transmisión de VIH es de 1: $600.000^{(18)}$ y de 26 en 1.000 .000 para infección bacteriana ${ }^{(16)}$.

El aloinjerto ofrece una amplia variedad de alternativas, con diversidad de tamaños, permitiendo elegir según la longitud, el grosor, la resistencia y el tipo de fijación (ósea o partes blandas). El tendón patelar y el tendón de Aquiles son los más utilizados, aunque aloinjertos de músculo tibial anterior, músculo tibial posterior y del tendón del músculo peroneo largo han demostrado una mayor resistencia biomecánica(19) (Figura 2).

El aloinjerto necesita mayor tiempo de incorporación y tiene menor resistencia biomecánica inicial comparado con los autoinjertos. Influye en esta menor resistencia el proceso de esterilización requerido para su uso. Rappe et al. ${ }^{(20)}$ demostraron un 33\% de fallos en el aloinjerto de tendón de Aquiles irradiado con 2 a 2,5 Mrad, comparado con el $2,4 \%$ del aloinjerto no irradiado. Se debe considerar además su disponibilidad y mayor costo.

\section{Injertos sintéticos}

Con el objetivo de encontrar un injerto ideal, se han desarrollado y utilizado injertos sintéticos. Estos pueden ser clasificados en matrices, stents o prótesis(21). Una matriz es fabricada a partir de biomateriales sintéticos (fibra de carbono, tereftalato de polietileno, etc.) que estimulan el crecimiento del tejido fibroso; a este grupo pertenece el LARS (ligament augmentation and reconstruction system). Los stents están pensados para proteger la consolidación de un injerto biológico durante su fase de incorporación; el LAD (Kennedy ligament augmentation device) es un buen ejemplo. Las prótesis son sustitutos del injerto biológico, fabricados con poliéster y GORE-TEX ${ }^{\circledR}$; en esta categoría encontramos implantes como el $\mathrm{ABC}$ (active biosynthetic composite, Surgicraft Ltd.). Desafortunadamente, estos dispositivos presentan una alta tasa de fracasos y complicaciones ${ }^{(22)}$. Las matrices de fibras de carbono se asocian a sinovitis, fracaso del crecimiento tisular e integración con los túneles óseos, con la subsecuente pérdida de las propiedades biomecánicas. En nuestro medio no se consideran como alternativa, ya que los utilizados previamente mostraron un alto grado de complicaciones, así como fracaso de la plastia.

\section{Resultados}

\section{Tendón patelar}

Se han publicado diversos estudios con un seguimiento mínimo de 5 años ${ }^{(3,23,24)}$ y han demostrado puntuaciones satisfactorias en las escalas de Lysholm, Tegner e IKDC mayores del 78 al 90\% de los 


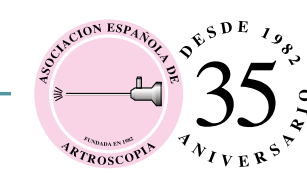

pacientes. Los mejores resultados funcionales se han visto en pacientes sin lesión meniscal al momento de la cirugía, alcanzando puntajes normales y cercanos a lo normal en el $90 \%$ de los pacientes. En ese grupo se demostró que el 98\% presentaba pivot shift de grado 0 y el $97 \%$ no presentaba cambios degenerativos al final del seguimiento ${ }^{(3,23)}$.

En un estudio de cohorte prospectivo con nivel de evidencia 2 realizado por Gifstadt et al. ${ }^{(25)}$, vieron que el tendón patelar presentaba una tasa de rerrotura significativamente menor que los isquiotibiales. Incluyeron 45.998 reconstrucciones primarias, observando que esta diferencia se mantenía al diferenciar según la edad y el género de los pacientes, y tendía a ser más significativa en deportes de pivote, como fútbol, balonmano o esquí alpino. Persson et al.(26), en otro estudio con nivel de evidencia 2, también demostraron que el autoinjerto isquiotibial presentaba el doble de riesgo de rerrotura al compararlo con el tendón patelar, principalmente en pacientes jóvenes.

\section{Tendones isquiotibiales}

Numerosos estudios han demostrado resultados comparables al tendón patelar. En un metaanálisis realizado por Freedman et al.(8), se encontró que, en un total de 1.976 pacientes, de 34 estudios, el autoinjerto de isquiotibiales presentaba una tasa de fracaso significativamente menor, con menor laxitud y mayor satisfacción del paciente al compararlo con el autoinjerto de tendón patelar. Sin embargo, en la bibliografía se encuentran datos contradictorios. Otro metaanálisis realizado por Yunes et al.(27), con un total de 411 pacientes, demostró que el autoinjerto de tendón patelar presentaba mejores resultados en laxitud residual al evaluarlos con KT-1000 ${ }^{\circledR}$ (MEDmetric, San Diego, CA) a 20 libras, y un mayor retorno deportivo al mismo nivel previo a la lesión. Este estudio no pudo determinar diferencias en cuanto a la morbilidad del sitio dador, al no contar con información comparable en los estudios incluidos. Una revisión sistemática ${ }^{(28)}$, realizada con estudios de nivel I y II, no mostró diferencias significativas en cuanto a fracasos y resultados de tendón patelar e isquiotibiales. El tendón patelar presentó mayor dolor anterior.

Se ha asociado el autoinjerto de los isquiotibiales a la debilidad flexora en el postoperatorio. Ardern et al. ${ }^{(29)}$ compararon 30 pacientes con autoinjerto de isquiotibiales con 20 pacientes en los que se tomó solo el músculo semitendinoso y con 32 meses promedio de seguimiento. No encontraron diferencias entre los grupos en cuanto a la medición de la fuerza isométrica e isocinética. En ambos grupos observaron un déficit del 3 al $27 \%$ en comparación con la extremidad no operada. Sin embargo, no observaron influencia en el retorno deportivo postoperatorio.

El fascículo cuádruple con tendones de los músculos semitendinoso y gracilis es el más resistente en laboratorio ${ }^{(11)}$. No obstante, esta ventaja biomecánica no siempre se traduce en resultados clínicos, especialmente en pacientes hiperlaxos. Kim et al. ${ }^{(12)}$ demostraron que los pacientes hiperlaxos, especialmente mujeres, presentaban mejores resultados clínicos y mayor estabilidad con el KT-1000 ${ }^{\circledR}$ cuando se realizaba una cirugía con autoinjerto de tendón patelar a 2 años de seguimiento.

\section{Tendón cuadricipital}

Existen series que muestran buenos resultados con tendón cuadricipital. Chen et al. ${ }^{(16)}$ describieron una serie de 12 pacientes con un seguimiento de 15 a 24 meses. Observaron que 10 pacientes retornaron a su nivel deportivo prelesional y 10 pacientes presentaron una puntuación en la escala de IKDC normal o cercano a lo normal. Sin embargo, después de un año, la fuerza del cuádriceps fue solo del $80 \%$ de la fuerza de la rodilla normal en 11 pacientes. En otro estudio, Lund et al. ${ }^{\left({ }^{30}\right)}$ compararon 25 pacientes con autoinjerto de tendón patelar y 26 pacientes con autoinjerto cuadricipital, con 1 a 2 años de seguimiento. No encontraron diferencias en el test de KT-1000 ${ }^{\circledR}$ ni en la escala del IKDC al final del seguimiento. Sin embargo, observaron que en el grupo de tendón cuadricipital fue visto con menor frecuencia un test de pivot shift positivo al final del seguimiento y que presentaban menos dolor anterior y menor pérdida sensitiva que el grupo de tendón patelar.

\section{Aloinjertos}

Diversos estudios han demostrado buenos resultados funcionales con aloinjertos y tasas de revisión de $5,45^{(31)}$ y $4,3 \%{ }^{(32)}$. Mascarehnas et al. ${ }^{(33)}$ realizaron un estudio comparativo en 19 pares de pacientes 
con autoinjerto de tendón patelar frente aloinjerto de tendón patelar; los pacientes practicaban deportes muy exigentes (fútbol, baloncesto) o exigentes (esquí, tenis), de 4 a 7 veces por semana, previamente a la lesión. Los resultados no demostraron diferencias en las escalas funcionales de IKDC, actividades de la vida diaria y puntaje de actividad deportiva del Knee Outcome Survey, ni en la forma corta del SF-36. Sin embargo, se observó que 14 pacientes del grupo de autoinjerto frente a 7 pacientes del grupo de aloinjerto reportaron ausencia de inestabilidad en pruebas muy exigentes. Además, 12 pacientes del grupo de autoinjerto retornaron a su nivel deportivo previo a la lesión y 10 pacientes del grupo de aloinjerto. Por otra parte, se debe cuidar el uso de aloinjerto en pacientes jóvenes activos, en los que se ha demostrado una mayor tasa de fracasos, hasta 4 veces mayor que en autoinjerto ${ }^{(34)}$. Borchers et al. ${ }^{(35)}$, en un estudio caso control con nivel de evidencia III, demostraron una tasa significativamente mayor en pacientes con actividad física de alta demanda y en los que se utilizó aloinjerto. Este riesgo se multiplicaba al interactuar ambas variables. Singhal et al. ${ }^{(36)}$ también demostraron una tasa de fracasos inaceptable (38\%) en una serie de 125 pacientes operados con aloinjerto de tendón tibial anterior, no recomendando su uso en pacientes menores de 25 años con actividad física de alta demanda. Además, se ha demostrado mayor laxitud residual del aloinjerto, en comparación con autoinjerto ${ }^{(37)}$. Su uso en revisiones también ha sido motivo de estudio. En el estudio multicéntrico de revisión de LCA (MARS)(38), se demostró que el aloinjerto tenía el doble de riesgo de rerrotura versus el autoinjerto (tendón patelar 0 isquiotibiales) y peores resultados en cuanto al retorno deportivo a 2 años de seguimiento.

\section{Recomendaciones}

Al seleccionar un injerto existen consideraciones biológicas y científicas; estas incluyen la edad, el tipo de deporte, la laxitud, el género, el peso y la talla; la incorporación en los túneles óseos hueso versus tejido blando; aloinjerto versus autoinjerto; la fijación ideal, las características psicológicas del paciente y sus expectativas. Además, se debe considerar el costo y las posibilidades económicas de cada paciente.

En los deportistas jóvenes, de alta demanda deportiva, ya sean profesionales o altamente compe- titivos que requieran un retorno deportivo precoz, recomendamos fuertemente el uso de tendón patelar, principalmente en deportes como el fútbol(25,26), el rugby, el fútbol americano y otros con riesgo de lesión de los isquiotibiales (carreras de velocidad). En deportes alpinos, como el esquí, el uso de tendón patelar ha demostrado menores riesgos de rerrotura ${ }^{(25)}$. Esta conducta está respaldada en los trabajos que muestran una incorporación más precoz del tendón patelar ${ }^{(5)}$, menor tasa de fallo de este, incluso en mujeres deportistas, y en los malos resultados del aloinjerto en pacientes jóvenes de alta demanda deportiva con una mayor tasa de rerrotura $^{(33-37)}$. Por otro lado, en otras actividades deportivas, con riesgo eventual de patología de tendón patelar, tales como el básquetbol, el voleibol y el tenis, el uso de autoinjerto de isquiotibiales es una excelente opción ${ }^{(21)}$. Sin embargo, se debe evaluar el nivel y las expectativas de cada paciente.

El autoinjerto de isquiotibiales ha demostrado resultados exitosos con buenas tasas de retorno deportivo en pacientes jóvenes (menores de 40 años) que practiquen deporte sin alta competencia ${ }^{(27)}$. Es importante el uso de un injerto de un diámetro adecuado (mayor de $8 \mathrm{~mm}$ ), por lo que el uso de técnicas de triplicado y quintuplicado puede ser una excelente opción en estos $\operatorname{casos}^{(39)}$. Este injerto es además la primera alternativa en pacientes con fisis abierta ${ }^{(40)}$ y en mujeres que necesiten una cirugía más estética. Sin embargo, se debe tener precaución al utilizarlo en pacientes hiperlaxos con hiperextensión de rodilla ${ }^{(12)}$. Recomendamos el uso de ortesis y un cauteloso protocolo de rehabilitación en estos pacientes. En pacientes mayores de 40 años, tanto el autoinjerto de isquiotibiales como el aloinjerto han demostrado buenos resultados. Nuestra tendencia es utilizar aloinjerto en pacientes de menor demanda o que quieran evitar sobremanera la morbilidad de la zona dadora. También recomendamos el uso de aloinjerto en lesiones multiligamentosas y en algunas revisiones. En el caso de revisiones en pacientes jóvenes, el uso de autoinjerto ha demostrado tener 2,78 veces menos riesgo de rerrotura subsecuente que el aloinjerto ${ }^{(37)}$.

Una mención especial pueden tener ciertas actividades profesionales como la danza y el levantamiento de pesas, en las que la pérdida tanto de la fuerza flexora como extensora puede comprometer su actividad. En estos pacientes, el uso de un aloinjerto con pastilla ósea también puede ser una opción viable, cuidando de cumplir los 


\begin{tabular}{|c|c|c|}
\hline Autoinjerto de tendón patelar & Autoinjerto de isquiotibiales & Aloinjerto \\
\hline $\begin{array}{l}\text { - Pacientes profesionales } \\
\text { y de alta competencia } \\
\text { (fútbol, rugby) } \\
\text { - Pacientes que requieran } \\
\text { retorno deportivo precoz } \\
\text { - Pacientes susceptibles de } \\
\text { lesión de isquiotibiales } \\
\text { (velocidad, fútbol } \\
\text { americano) } \\
\text { - Pacientes hiperlaxos }\end{array}$ & $\begin{array}{l}\text { - Pacientes con fisis abiertas } \\
\text { - Mujeres (ventaja estética) } \\
\text { - Pacientes que requieran } \\
\text { arrodillarse } \\
\text { - Deportes susceptibles de } \\
\text { daño al tendón patelar } \\
\text { (básquetbol, voleibol, } \\
\text { tenis) }\end{array}$ & $\begin{array}{l}\text { - Lesiones } \\
\text { multiligamentosas } \\
\text { - Pacientes mayores de } 40 \\
\text { años de baja demanda } \\
\text { - Revisiones, no jóvenes de } \\
\text { alta demanda }\end{array}$ \\
\hline
\end{tabular}

6. Jackson DW, Grood ES, Goldstein JD, Rosen MA, Kurzweil PR, Cummings $J F$, et al. A comparison of patellar tendon autograft and allograft used for anterior cruciate ligament reconstruction in the goat model. Am J Sports Med. 1993;21:176-85.

7. Brown $\mathrm{CH}$. $\mathrm{ACL}$ Surgery. Graft options: patellar tendon, hamstrings tendons, quadriceps tendon and allografts. En: Sim FH (ed.). American Academy

tiempos de rehabilitación para no comprometer la integración del injerto (Tabla 3).

Basándose en la bibliografía, el autoinjerto parece ser superior al aloinjerto con respecto a la tasa de rerrotura. No existen diferencias entre las opciones de autoinjerto de tendón patelar o isquiotibiales, ya que la literatura actual no apoya consistentemente una opción sobre la otra. Se requieren estudios de mayor evidencia que consideren la edad y la actividad del paciente para definir con mayor claridad un tipo de injerto específico en cada disciplina deportiva y grupo etario.

\section{Bibliografía}

1. Prodromos CC, Fu FH, Howell SM, Johnson DH, Lawhorn K. Controversies in soft-tissue anterior cruciate ligament reconstruction: grafts, bundles, tunnels, fixation, and harvest. J Am Acad Orthop Surg. 2008;16:376-84.

2. Duchman KR, Lynch TS, Spindler KP. Graft Selection in Anterior Cruciate Ligament Surgery. Who gets what and why? Clin Sports Med. 2017;36:25-33.

3. Deehan DJ, Salman LJ, Webb VJ, Davies A, Pinczewski LA. Endoscopic reconstruction of the anterior cruciate ligament with an ipsilateral patellar tendon autograft: a prospective longitudinal five-year study. J Bone Joint Surg (Br). 2000;82-B:984-91.

4. Jarvela T, Nyyssonen M, Kannus P, Paakkala T, Järvinen M. Bone patellar tendon bone reconstruction for the anterior cruciate ligament: a long term comparison of early and late repair. Int Orthop. 1999;23:227-31.

5. West RV, Harner CD. Graft selection in anterior cruciate ligament reconstruction. J Am Acad Orthop Surg. 2005;13:197-207. of Ortopaedic Surgeons 2001 instructional course lectures. Park Ridge, IL: American Academy of Orthopaedic Surgeons; 2001. pp. 447-58.

8. Freedman KB, D'Amato MJ, Nedeff DD, Kaz A, Bach BR Jr. Arthroscopic anterior cruciate ligament reconstruction: a meta-analysis comparing patellar tendon and hamstring tendon autografts. Am J Sports Med. 2003;31:2-11.

9. Shelton WR, Papendick L, Dukes AD. Autograft versus allograft anterior cruciate ligament reconstruction. Arthroscopy. 1997;13:446-9.

10. Lephart SM, Kocher MS, Harner CD, Fu FH. Quadriceps strength and functional capacity after anterior cruciate ligament reconstruction: patellar tendon autograft versus allograft. Am J Sports Med. 1993;21:738-43.

11. Hamner DL, Brown CH Jr, Steiner ME, Hecker AT, Hayes WC. Hamstring tendon grafts for reconstruction of the anterior cruciate ligament: biomechanical evaluation of the use of multiple strands and tensioning techniques. J Bone Joint Surg (Am). 1999;81-A:549-57.

12. Kim SJ, Kim TE, Lee DH, Oh KS. Anterior cruciate ligament reconstruction in patients who have excessive joint laxity. J Bone Joint Surg (Am). 2008;90:735-41.

13. Stäubli HU, Schatzmann L, Brunner P, Rincón L, Nolte LP. Mechanical tensile properties of the quadriceps tendon and patella ligament in young adults. Am J Sports Med. 1999;27:27-34.

14. Fulkerson JP, Langeland R. An alternative cruciate reconstruction graft : the central quadriceps tendon. Arthroscopy. 1995;11:252-4.

15. Fu FH. Anterior cruciate ligaments: graft selection in 2009. Instructional Course Lecture 309, AAOS Annual Meeting; 2009.

16. Chen $\mathrm{CH}$, Chen WJ, Shih $\mathrm{CH}$. Arthroscopic anterior cruciate ligament reconstruction with quadriceps tendon patellar bone autograft. J Trauma. 1999;46:678-82. 
17. Simonds RJ, Holmberg SD, Hurwitz RL, Coleman TR, Bottenfield S, Conley LJ, et al. Transmission of human immunodeficiency virus type 1 from a seronegative organ and tissue donor. N Engl J Med. 1992;326:726-32.

18. Buck BE, Malinin TI, Brown MD. Bone transplantation and human immunodeficiency virus: an estimated risk of acquired immunodeficiency syndrome (AIDS). Clin Orthop Rel Res. 1989;240:129-36.

19. Pearsall AW 4th, Hollis JM, Russell GV Jr, Scheer Z. A biomechanical comparison of three lower extremity tendons for ligamentous reconstruction about the knee. Arthroscopy. 2003;19:1091-6.

20. Rappe M, Horodyski M, Meister K, Indelicato PA. Nonirradiated versus irradiated Achilles allograft: in vivo failure comparison. Am J Sports Med. 2007;35:1653-8.

21. Bonasia DE, Amendola A. Graft choice in $A C L$ reconstruction. En: Bonnin M, Amendola NA, Bellemans J, MacDonald SJ, Menetrey J (eds.). The Knee Joint. Surgical Techniques and Strategies. Springer; 2012: 173-181.

22. Iliadis DP, Bourlos DN, Mastrokalos DS, Chronopoulos E, Babis GC. LARS artificial ligament versus abc purely polyester ligament for anterior cruciate ligament reconstruction. Orthop J Sports Med. 2016;4:2325967116653359.

23. Bartlett RJ, Clatworthy MG, Nguyen TN. Graft selection in reconstruction of the anterior cruciate ligament. J Bone Joint Surg (Br). 2001;83-B:625-34.

24. Shelbourne KD, Gray T. Results of anterior cruciate ligament reconstruction based on meniscus and articular cartilage status at the time of surgery. Am J Sports Med. 2000;28:446-52.

25. Gifstad T, Foss OA, Engebretsen L, Lind M, Forssblad M, Albrektsen $G$, et al. Lower risk of revision with patellar tendon autografts compared with hamstring autografts: a registry study based on 45,998 primary ACL reconstructions in Scandinavia. Am J Sports Med. 2014;42:2319-28.

26. Persson A, Fjeldsgaard K, Gjertsen JE, Kjellsen AB, Engebretsen L, Hole RM, et al. Increased risk of revision with hamstring tendon grafts compared with patellar tendon grafts after anterior cruciate ligament reconstruction: a study of 12,643 patients from the Norwegian Cruciate Ligament Registry, 2004-2012. Am J Sports Med. 2014;42:285-91.

27. Grana WA. Re: "Patellar versus hamstring tendons in anterior cruciate ligament reconstruction: A meta-analysis" by Yunes et al. in the March 2001 issue. Arthroscopy. 2001;17:791-2.

28. Ciccotti MC, Secrist E, Tjoumakaris F, Ciccotti MG, Freedman KB. Anatomic Anterior Cruciate Ligament Reconstruction via Independent Tunnel Drilling: A Systematic Review of Randomized Controlled Trials Comparing Patellar Tendon and Hamstring Autografts. Arthroscopy. 2017. [Epub ahead of print].
29. Ardern CL, Webster KE, Taylor NF, Feller JA. Hamstring strength recovery after hamstring tendon harvest for anterior cruciate ligament reconstruction: a comparison between graft types. Arthroscopy. 2010;26:462-9.

30. Lund B, Nielsen T, Faun $\phi$ P, Christiansen SE, Lind M. IS quadriceps tendon a better graft choice than patellar tendon? a prospective randomized study. Arthroscopy. 2014;30:593-8.

31. Almqvist KF, Willaert P, De Brabandere S, Criel K, Verdonk R. A long-term study of anterior cruciate ligament allograft reconstruction. Knee Surg Sports Traumatol Arthrosc. 2009;17:818-22.

32. Edgar CM, Zimmer S, Kakar S, Jones H, Schepsis AA. Prospective comparison of auto and allograft hamstring tendon constructs for $\mathrm{ACL}$ reconstruction. Clin Orthop Relat Res. 2008;466:2238-46.

33. Mascarenhas R, Tranovich M, Karpie JC, Irrgang JJ, Fu FH, Harner CD. Patellar tendon anterior cruciate ligament reconstruction in the high-demand patient: evaluation of autograft versus allograft reconstruction. Arthroscopy. 2010;26(Suppl):58-66.

34. Kaeding CC, Aros B, Pedroza A, Pifel E, Amendola A, Andrish JT, et al. Allograft Versus Autograft Anterior Cruciate Ligament Reconstruction: Predictors of Failure from a MOON Prospective Longitudinal Cohort. Sports Health. 2011;3:73-81.

35. Borchers JR, Pedroza A, Kaeding C. Activity level and graft type as risk factors for anterior cruciate ligament graft failure. Am J Sports Med. 2009;37:2362-7.

36. Singhal MC, Gardiner JR, Johnson DL. Failure of primary anterior cruciate ligament surgery using anterior tibialis allograft. Arthroscopy. 2007;23:469-75.

37. Prodromos C, Joyce B, Shi K. A meta-analysis of stability of autografts compared to allografts after anterior cruciate ligament reconstruction. Knee Surg Sports Traumatol Arthrosc. 2007;15:851-6.

38. MARS Group. Effect of graft choice on the outcome of revision anterior cruciate ligament reconstruction in the Multicenter ACL Revision Study (MARS) Cohort. Am J Sports Med. 2014;42:2301-10.

39. Calvo R, Figueroa D, Figueroa F, Vaisman A, Schmidt-Hebbel A, Morales N, et al. Five Strand Hamstring Autograft Versus Quadruple Hamstring Autograft With Graft Diameters 8.0 Millimeters or More in Anterior Cruciate Ligament Reconstruction: Clinical Outcomes With a Minimum 2-Year Follow-Up. Arthroscopy. 2017. [Epub ahead of print].

40. Calvo R, Figueroa D, Gili F, Vaisman A, Mocoçain P, Espinosa M, et al. Transphyseal anterior cruciate ligament reconstruction in patients with open physes: 10-year follow-up study. Am J Sports Med. 2015;43:289-94. 